\title{
Monotone schemes for time-dependent energy balance models
}

\author{
Roderick V. N. Melnik* Janis Rimshans ${ }^{\dagger}$
}

(Received 8 August 2003; revised 20 January 2004)

\begin{abstract}
In this paper we derive a new model for the description of fast transient processes in optically sensitive semiconductors. Based on the generalised flow approach, we develop a new difference scheme for this energy balance model and show that the solution of the derived scheme satisfies the maximum principle. The model is applicable to the analysis of photodevices with fast response, and is particular useful in estimating a photo-charge response in the depletion region of phototransistor semiconductor structures.
\end{abstract}

*Syddansk Universitet, MCI, Faculty of Science \& Engineering, DK-6400, Denmark. mailto:rmelnik@mci.sdu.dk

${ }^{\dagger}$ University of Latvia, Institute of Mathematics and Computer Science, LV-1459, LATVIA.

See http://anziamj.austms.org.au/V45/CTAC2003/Meln/home.html for this article, (c) Austral. Mathematical Soc. 2004. Published July 29, 2004. ISSN 1446-8735 


\section{Contents}

1 Introduction

C730

2 Governing equations for fast processes in optically sensitive semiconductors

C732

3 Construction of monotone numerical approximations

C736

4 Numerical results

C739

References

C742

\section{Introduction}

Monotone conservative numerical approximations play a fundamental role in most applications of mathematical modelling tools to problems in science and engineering. In this paper our major interest is to derive a model and a monotone conservative scheme for its solution that would allow us to describe nonlinear transport phenomena in semiconductor structures with fast photoresponse. Such optically sensitive devices are gaining an increasing popularity in many engineering applications [7], while for a range of already existing devices, including HEBT (hetero-structure-emitter bipolar transistors) and VFEPT (vertical field-effect photo-transistors), these transport phenomena are at the heart of functionality of the devices [1].

Although the development of efficient methodologies for constructing monotone numerical approximations for strongly nonlinear coupled systems of partial differential equations is extremely important for many applications, including semiconductor device theory, it requires overcoming a number of well known and highly non-trivial difficulties. Recall that classical explicit schemes, such as the Lax-Wendroff scheme, are not monotone (in addition 
to quite restrictive stability conditions of the CFL type). When made monotone, such schemes typically lose the conservation properties [9, e.g.]. To resort in such cases to upwind-type approximations may not always be a real remedy due to quite restrictive conditions for such schemes in order to be monotone, in particular in the case of variable convection coefficients. On the other hand, classical implicit schemes, such as the standard CrankNicolson time stepping, are also non-monotone in general and it has been shown that for nonlinear convection-diffusion models these schemes can converge to incorrect solutions, or lead to instability [5, e.g.]. New interesting results appeared recently in the context of generalisations of the MarkowichZlamal approach (originally proposed for symmetrisable models) which leads to classical approximations of the Scharfetter-Gummel type [8]. However, the discussion has been limited to a model convection-diffusion equation in time-independent situations.

In the present study we consider a half-implicit, monotone, exponential, non-linear scheme with the aim of solving a system of coupled PDEs describing fast transport phenomena in optically sensitive semiconductors. The major thread of our discussion will be centred around an unsteady, non-symmetrisable energy balance equation. The paper is organized as follows. In Section 2 we formulate a general system of equations describing carrier transport in semiconductor structures illuminated by the monochromatic light. Based on this system, we derive a simplified model that allows us to study fast transport phenomena. In Section 3 we derive a new difference scheme for our model and show that its solution satisfies the maximum principle. Results of computational experiments are discussed in Section 4. 


\section{Governing equations for fast processes in optically sensitive semiconductors}

In what follows, we are interested in the description of transient processes in a semiconductor structure illuminated by the monochromatic light. In such structures, one typically observes two groups of carriers, conductive and photogenerated. We assume that the structure is in the dynamic equilibrium with respect to concentrations of conductive electrons $\left(n_{c}\right)$ and holes $\left(p_{c}\right)$, and the concentrations of photogenerated (or "excess") charges (denoted here as $n_{e}$ and $p_{e}$ ) are large enough to introduce electron and holes temperatures in the semiconductor system (denoted as $T_{n}^{c}, T_{n}^{e}$ and $T_{p}^{c}, T_{p}^{e}$, respectively). We use a generic sub-index " $\alpha$ " indicating conductive carriers when $\alpha=c$ and photogenerated carriers when $\alpha=e$. Using this notation, our semiconductor system is described by the following system of strongly coupled nonlinear PDES:

$$
\begin{aligned}
& \frac{\partial n_{\alpha}}{\partial t}-\frac{\partial J_{n}^{\alpha}}{\partial x}=-\left(R^{\alpha}-G^{\alpha}\right), \\
& \frac{3}{2} \frac{\partial}{\partial t}\left(n_{\alpha} T_{n}^{\alpha}\right)+\frac{\partial}{\partial x} S_{n}^{\alpha}=-J_{n}^{\alpha} \frac{\partial \varphi}{\partial x}+P_{n}^{\alpha}, \\
& \frac{\partial p_{\alpha}}{\partial t}+\frac{\partial J_{p}^{\alpha}}{\partial x}=-\left(R^{\alpha}-G^{\alpha}\right), \\
& \frac{3}{2} \frac{\partial}{\partial t}\left(p_{\alpha} T_{p}^{\alpha}\right)+\frac{\partial}{\partial x} S_{p}^{\alpha}=-J_{p}^{\alpha} \frac{\partial \varphi}{\partial x}+P_{p}^{\alpha}, \\
& \frac{\partial}{\partial x}\left(\kappa \frac{\partial \varphi}{\partial x}\right)=n_{c}-p_{c}+n_{e}-p_{e}-\left(N_{d}-N_{a}\right),
\end{aligned}
$$

with fluxes for the carrier densities,

$$
\begin{aligned}
J_{n}^{\alpha} & =-\mu_{n}^{\alpha} n_{\alpha} \frac{\partial \varphi}{\partial x}+\frac{\partial}{\partial x}\left(\mu_{n}^{\alpha} n_{\alpha} T_{n}^{\alpha}\right) \\
J_{p}^{\alpha} & =-\mu_{p}^{\alpha} p_{\alpha} \frac{\partial \varphi}{\partial x}-\frac{\partial}{\partial x}\left(\mu_{p}^{\alpha} p_{\alpha} T_{p}^{\alpha}\right)
\end{aligned}
$$


and energy fluxes,

$$
\begin{aligned}
S_{n}^{\alpha} & =-C_{e}^{\alpha}\left(-\mu_{n}^{\alpha} n_{\alpha} \frac{\partial \varphi}{\partial x} T_{n}^{\alpha}+\frac{\partial}{\partial x}\left(\mu_{n}^{\alpha} n_{\alpha}\left(T_{n}^{\alpha}\right)^{2}\right)\right), \\
S_{p}^{\alpha} & =-C_{h}^{\alpha}\left(\mu_{p}^{\alpha} p_{\alpha} \frac{\partial \varphi}{\partial x} T_{p}^{\alpha}+\frac{\partial}{\partial x}\left(\mu_{p} p_{\alpha}\left(T_{p}^{\alpha}\right)^{2}\right)\right) .
\end{aligned}
$$

The above system is considered in the space-time region $\bar{\Gamma}=\{(x, t) \mid 0 \leq$ $x \leq L, 0 \leq t \leq T\}$. Notations used here are standard, and we remind for completeness that $\varphi$ is the electrostatic potential (so that $E=-\partial \varphi / \partial x$ is the electrostatic field), $\mu_{n}^{\alpha}$ and $\mu_{p}^{\alpha}$ are mean electron and holes mobilities, $C_{e}^{\alpha}$ and $C_{h}^{\alpha}$ are Peltier's coefficients for electrons and holes, respectively. As usual, $R^{\alpha}$ and $G^{\alpha}$ denote recombination and generation terms, respectively (we assume that $G^{c} \equiv 0$ ), $\kappa$ is the dielectric constant of the semiconductor material, $N_{d}$ and $N_{a}$ are doping concentrations (donor and acceptor, respectively), and $P_{n}^{\alpha}, P_{p}^{\alpha}$ are electron and holes power densities that are responsible for the rate of energy losses induced by scattering on the lattice and other dissipative effects. The system (1-9) is given in a normalised form, with the normalisation constants chosen in a way analogous to that in [3]. The structure of interest here is a VFEPT considered and analysed previously with simplified models $\left[1,4\right.$, e.g.]. Boundary conditions for concentrations $c_{\alpha}$ (either $n_{\alpha}$ or $p_{\alpha}$ ) and for the energy densities $\epsilon^{\alpha}=3 / 2 c_{\alpha} T_{\alpha}$ are

$$
\begin{array}{ll}
\eta_{1}^{\alpha} \frac{\partial}{\partial x} c_{\alpha}(x, t)-\sigma_{1}^{\alpha} c_{\alpha}(x, t)=f_{1}^{\alpha}, & \text { on } x=0, L, \\
\eta_{2}^{\alpha} \frac{\partial}{\partial x} \epsilon^{\alpha}(x, t)-\sigma_{2}^{\alpha} \epsilon^{\alpha}(x, t)=f_{2}^{\alpha}, & \text { on } x=0, L,
\end{array}
$$

with given $\eta_{i}^{\alpha}, \sigma_{i}^{\alpha}$, and $f_{i}^{\alpha}, i=1,2$, while for the potential, Dirichlet's boundary conditions are assumed. Initial conditions for concentrations $\left(c_{\alpha}\right)$, energy densities $\left(\epsilon^{\alpha}\right)$, and potential $(\varphi)$ are

$$
c_{\alpha}(x, 0)=c_{0}^{\alpha}(x), \quad \epsilon^{\alpha}(x, 0)=\epsilon_{0}^{\alpha}(x), \quad \varphi(x, 0)=\varphi_{0}(x),
$$

with given initial distributions $c_{0}^{\alpha}(x), \epsilon_{0}^{\alpha}(x)$, and $\varphi_{0}(x)$. 
One of the characteristics of major importance for such devices is their (photo)response time, or, to put it differently, a time needed to reach a steady-state photocurrent. We are interested in modelling fast transient processes related to photoresponse phenomena which are phenomena of principal interest in the depletion region of VFEPT structures. It appears that these phenomena can be adequately described by a system which is substantially simpler compared to our original model (1-12). The main idea behind such a simplification lies with the fact that charge transport processes can be subdivided into fast and slow. We start from the analysis of continuity equations (1) and (3) and consider their sum, and then their difference: the sum gives

$$
\frac{\partial N_{\alpha}}{\partial t}-\frac{\partial}{\partial x} \delta F^{\alpha}=-2\left(R^{\alpha}-G^{\alpha}\right),
$$

with $N_{\alpha}=n_{\alpha}+p_{\alpha}$ and with the flux difference

$$
\begin{aligned}
\delta F^{\alpha} \equiv & J_{n}^{\alpha}-J_{p}^{\alpha}=-\left(\mu_{n}^{\alpha} n_{\alpha}-\mu_{p}^{\alpha} p_{\alpha}\right) \frac{\partial \varphi}{\partial x} \\
& -\frac{\partial}{\partial x}\left(\mu_{n}^{\alpha} n_{\alpha} T_{p}^{\alpha}+\mu_{p}^{\alpha} p_{\alpha} T_{n}^{\alpha}\right)+\frac{\partial}{\partial x}\left\{\left(\mu_{n}^{\alpha} T_{n}^{\alpha}+\mu_{p}^{\alpha} T_{p}^{\alpha}\right) N_{\alpha}\right\}
\end{aligned}
$$

similarly, the difference of the continuity equations gives

$$
\frac{\partial \delta n_{\alpha}}{\partial t}-\frac{\partial}{\partial x} F^{\alpha}=0
$$

with $\delta n_{\alpha}=n_{\alpha}-p_{\alpha}$ and the sum of the fluxes

$$
\begin{aligned}
F^{\alpha} \equiv & J_{n}^{\alpha}+J_{p}^{\alpha}=-\left(\mu_{n}^{\alpha} n_{\alpha}+\mu_{p}^{\alpha} p_{\alpha}\right) \frac{\partial \varphi}{\partial x}+\mu_{n}^{\alpha} T_{n}^{\alpha} \frac{\partial p_{\alpha}}{\partial x}-\mu_{p}^{\alpha} T_{p}^{\alpha} \frac{\partial n_{\alpha}}{\partial x} \\
& +\frac{\partial}{\partial x}\left(\mu_{n}^{\alpha} T_{n}^{\alpha}\right) n_{\alpha}-\frac{\partial}{\partial x}\left(\mu_{p}^{\alpha} T_{p}^{\alpha}\right) p_{\alpha}+\left(\mu_{n}^{\alpha} T_{n}^{\alpha}+\mu_{p}^{\alpha} T_{p}^{\alpha}\right) \frac{\partial}{\partial x} \delta n_{\alpha} \cdot(16)
\end{aligned}
$$

Clearly, given the solutions to (13) and (15), the values of the concentrations $n_{\alpha}$ and $p_{\alpha}$ can be reconstructed as

$$
n_{\alpha}=\frac{1}{2}\left(N_{\alpha}+\delta n_{\alpha}\right) \quad \text { and } \quad p_{\alpha}=\frac{1}{2}\left(N_{\alpha}-\delta n_{\alpha}\right) .
$$


Note now that the system of equations (15-16) does not contain a recombination-generation term, and furthermore, the drift term in the flux expression (16) is substantially larger compared to the corresponding term in the equation (14), in particular in high electric fields. Hence, for a sufficiently large electric field and mobilities of carriers, the equation (15) supplemented by (16) describes a fast part of the charge transfer process, that is mainly the drift part. Next, if we assume that in the initial state, electron and hole temperatures are equal $\left(T_{n}^{\alpha}=T_{p}^{\alpha}=T^{\alpha}\right)$, and that during the fast stage of charge transfer they remain sufficiently close to each other, we apply arguments similar to that already discussed above to conclude that the sum of equations (2) and (4) provide the second equation to our system aimed at the description of fast processes:

$$
\frac{\partial}{\partial t}\left(\epsilon^{\alpha}\right)+\frac{\partial}{\partial x} S^{\alpha}=-F^{\alpha} \frac{\partial \varphi}{\partial x}+P^{\alpha},
$$

where $\epsilon^{\alpha}=3 / 2 N_{\alpha} T^{\alpha}$. For simplicity, we assume that $\mu_{n}=\mu_{p}=\mu$ and $C_{e}^{\alpha}=C_{h}^{\alpha}=C^{\alpha}$, in which case the flux energy

$$
S^{\alpha}=-C^{\alpha}\left(-\mu^{\alpha} \frac{\partial \varphi}{\partial x}\left(\frac{2}{3} \epsilon^{\alpha}-2 p^{\alpha} T^{\alpha}\right)+\frac{2}{3} \frac{\partial}{\partial x}\left(\mu T^{\alpha} \epsilon^{\alpha}\right)\right) .
$$

The term $P^{\alpha}=P_{n}^{\alpha}+P_{p}^{\alpha}$ in the right hand side of (17) is approximated with the time-relaxation approximation $P^{\alpha}=\left(N_{\alpha} T_{l}-2 / 3 \epsilon^{\alpha}\right) / \tau_{\omega}$, where $T_{l}$ is the lattice temperature and $\tau_{\omega}$ is the energy relaxation time. To proceed to the solution of system (15-18) coupled with Poisson's equation (5) we need an approximation for $N_{\alpha}$. This is obtained by assuming that the value of $N_{\alpha}$ is not influenced by diffusion and recombination processes during the stage of fast charge transfer, in which case we use $N_{\alpha}=N_{\alpha}^{0}+\delta n_{\alpha}$ with $N_{\alpha}^{0}$ taken as the initial state value of this concentration. 


\section{Construction of monotone numerical approximations}

In [4] we succeeded in constructing monotone conservative schemes for the analysis of the VFEPT devices for the constant temperature case, but the task of generalising those ideas to our current model is non-trivial. This is due to changeability of the sign in the term $-F^{\alpha} \partial \varphi / \partial x$ of equation (17) which typically leads to breaking up monotonicity conditions for traditional numerical techniques.

We explain our technique taking equation (17) as an example, not only due to the limited space, but also because approximating this equation is the most challenging task when approximating the entire system (15-18) and (5). First, we re-write this equation as

$$
\frac{\partial}{\partial t}\left(\epsilon^{\alpha}\right)+\frac{\partial}{\partial x} I^{\alpha}=P^{\alpha}
$$

where $I^{\alpha}$ is the generalized energy flux density defined as follows

$$
\begin{aligned}
I^{\alpha}= & -\frac{2}{3} C^{\alpha} \mu^{\alpha} T^{\alpha} \frac{\partial}{\partial x}\left(\epsilon^{\alpha}\right)+\frac{2}{3} C^{\alpha}\left(\mu^{\alpha} \frac{\partial \varphi}{\partial x}-\frac{\partial}{\partial x}\left(\mu^{\alpha} T^{\alpha}\right)\right) \epsilon^{\alpha} \\
& -2 C^{\alpha} \mu^{\alpha} p_{\alpha} T^{\alpha} \frac{\partial}{\partial x} \varphi+\frac{2}{3} \int_{x_{0}}^{x} d x v^{\alpha} \frac{\partial}{\partial x}\left(\epsilon^{\alpha}\right),
\end{aligned}
$$

with $x_{0}$ being a real number, and

$$
\begin{aligned}
v^{\alpha}=\frac{3}{2} & \frac{\partial}{\partial x} \varphi\left(\frac{\partial}{\partial x}\left(\epsilon^{\alpha}\right)\right)^{-1}\left(\frac{2}{3} \epsilon^{\alpha} \frac{\partial}{\partial x} \mu^{\alpha}-\mu^{\alpha} N_{\alpha} \frac{\partial}{\partial x} \varphi\right. \\
+ & \left.\frac{\partial}{\partial x}\left(2 p_{\alpha} T^{\alpha}\right)\right)+\mu^{\alpha} \frac{\partial}{\partial x} \varphi
\end{aligned}
$$

being interpreted as the velocity of the process. On a nonuniform grid $\omega_{\tau h}=$ $\omega_{\tau} \times \omega_{h}$ with $\left.\omega_{\tau}=\left\{\tau_{l+1}=\left(t_{l+1}-t_{l}\right) \mid l=0, \ldots, M_{t}-1\right)\right\}$ and $\omega_{h}=\left\{h_{i+1}=\right.$ 
$\left.\left.\left(x_{i+1}-x_{i}\right) \mid i=0, \ldots, M_{x}-1\right)\right\}$, covering the region $\bar{\Gamma}$, we apply the integrointerpolation method $[6,3]$ to get the following discretisation for (19):

$$
\frac{\left(\epsilon^{\alpha}\right)_{i}^{l+1}-\left(\epsilon^{\alpha}\right)_{i}^{l}}{\tau_{l+1}}+\frac{\left(I^{\alpha}\right)_{i+1 / 2}^{l+1}-\left(I^{\alpha}\right)_{i-1 / 2}^{l+1}}{h_{i}^{*}}=\frac{\left(N_{\alpha} T_{l}\right)_{i}-2 / 3\left(\epsilon^{\alpha}\right)_{i}^{l+1}}{\tau_{\omega}}
$$

for $1 \leq i \leq M_{x}-1,0 \leq l \leq M_{t}-1$, where $\left(I^{\alpha}\right)_{i \pm 1 / 2}$ are the averaged fluxes on the grid points and $h_{i}^{*}=\frac{1}{2}\left(h_{i+1}+h_{i}\right)$. In order to obtain the averaged values of the grid fluxes $\left(I^{\alpha}\right)_{i+1 / 2}$, we introduce the following exponential substitution for $\epsilon^{\alpha}$

$$
\begin{aligned}
\epsilon^{\alpha}= & \Omega^{\alpha}(x, t) \exp \left(I_{o}^{\alpha}\left(x_{0}, x\right)\right), \quad I_{o}^{\alpha}\left(x_{b}, x_{f}\right)=\int_{x_{b}}^{x_{f}} d x \omega(x), \\
\omega(x)= & \frac{1}{\mu^{\alpha} T^{\alpha}}\left(\mu^{\alpha} \frac{\partial \varphi}{\partial x}-\frac{\partial}{\partial x}\left(\mu^{\alpha} T^{\alpha}\right)\right)-\frac{3}{\epsilon^{\alpha}} p_{\alpha} \frac{\partial}{\partial x} \varphi \\
& +\frac{1}{C^{\alpha} \mu^{\alpha} T^{\alpha} \epsilon^{\alpha}} \int_{x_{0}}^{x} d x v^{\alpha} \frac{\partial}{\partial x}\left(\epsilon^{\alpha}\right) .
\end{aligned}
$$

Using (23) for the energy density, the flux (20) is written in the divergencelike form

$$
I^{\alpha}=-C^{\alpha} \mu^{\alpha} T^{\alpha} \exp \left(I_{o}^{\alpha}\left(x_{0}, x\right)\right) \frac{\partial}{\partial x} \Omega^{\alpha} .
$$

Integrating the flux (25) over the segment $x \in\left[x_{i}, x_{i+1}\right]$ and assuming that $I^{\alpha}$ takes the constant values $I^{\alpha}=\left(I^{\alpha}\right)_{i+1 / 2}$ within this segment, we obtain

$$
\begin{aligned}
\left(I^{\alpha}\right)_{i+1 / 2} & =-\frac{\left(\Omega^{\alpha}\right)_{i+1}-\left(\Omega^{\alpha}\right)_{i}}{I_{d}^{\alpha}\left(x_{i}, x_{i+1}\right)} \\
I_{d}^{\alpha}\left(x_{b}, x_{f}\right) & =\int_{x_{b}}^{x_{f}} d x \frac{1}{C^{\alpha} \mu^{\alpha} T^{\alpha}} \exp \left(-I_{o}^{\alpha}\left(x_{b}, x\right)\right) .
\end{aligned}
$$

Replacing $\Omega^{\alpha}$ in (26) by $\epsilon^{\alpha}$ from (23), we have

$$
\left(I^{\alpha}\right)_{i+1 / 2}=-\frac{2}{3} \frac{\exp \left(-I_{o}^{\alpha}\left(x_{i}, x_{x+1}\right)\right)\left(\epsilon^{\alpha}\right)_{i+1}-\left(\epsilon^{\alpha}\right)_{i}}{I_{d}^{\alpha}\left(x_{i}, x_{i+1}\right)} .
$$


Under similar assumptions for the interpolated values involving $\omega^{\alpha}(x)$ (defined in the same way as (24)), we finally arrive at the approximation

$$
\left(I^{\alpha}\right)_{i+1 / 2}=-\frac{2}{3} C^{\alpha}\left(\mu^{\alpha} T^{\alpha}\right)_{i+1 / 2}\left(\beta_{\epsilon}^{\alpha}\right)_{i+1 / 2} \frac{\left(\epsilon^{\alpha}\right)_{i+1}-\exp \left(\left(\beta_{\epsilon}^{\alpha}\right)_{i+1 / 2}\right)\left(\epsilon^{\alpha}\right)_{i}}{h_{i+1}\left(\exp \left(\left(\beta_{\epsilon}^{\alpha}\right)_{i+1 / 2}\right)-1\right)}
$$

where

$$
\begin{aligned}
& \left(\beta_{\epsilon}^{\alpha}\right)_{i+1 / 2}=\frac{1}{T_{i+1 / 2}^{\alpha}}\left(\varphi_{i+1}-\varphi_{i}\right)-\frac{1}{\left(\mu^{\alpha} T^{\alpha}\right)_{i+1 / 2}}\left(\left(\mu^{\alpha} T^{\alpha}\right)_{i+1}-\left(\mu^{\alpha} T^{\alpha}\right)_{i}\right) \\
& -\frac{3\left(p^{\alpha}\right)_{i+1 / 2}}{\left(\epsilon^{\alpha}\right)_{i+1 / 2}}\left(\varphi_{i+1}-\varphi_{i}\right)+\frac{3}{2} \frac{\xi_{i}^{\alpha} h_{i+1}}{\left(C^{\alpha} \mu^{\alpha} T^{\alpha} \epsilon^{\alpha}\right)_{i+1 / 2}}+\frac{3}{8\left(C^{\alpha} \mu^{\alpha} T^{\alpha} \epsilon^{\alpha}\right)_{i+1 / 2}} \\
& \times\left\{\left[\frac{2}{3} \epsilon_{i+1 / 2}^{\alpha}\left(\mu_{i+1}^{\alpha}-\mu_{i}^{\alpha}\right)-\left(\mu^{\alpha} N_{\alpha}\right)_{i+1 / 2}\left(\varphi_{i+1}-\varphi_{i}\right)-2 \mu_{i+1 / 2}^{\alpha}\left(\left(p^{\alpha} T^{\alpha}\right)_{i+1}\right.\right.\right. \\
& \left.\left.\left.\quad-\left(p^{\alpha} T^{\alpha}\right)_{i}\right)\right]\left(\varphi_{i+1}-\varphi_{i}\right)+\frac{2}{3} \mu_{i+1 / 2}^{\alpha}\left(\varphi_{i+1}-\varphi_{i}\right)\left(\epsilon_{i+1}^{\alpha}-\epsilon_{i}^{\alpha}\right)\right\}
\end{aligned}
$$

and

$$
\xi_{i}^{\alpha}=\frac{2}{3} \sum_{n=0}^{i-1} v_{n+1 / 2}^{\alpha}\left(\epsilon_{n+1}^{\alpha}-\epsilon_{n}^{\alpha}\right), \quad a_{i+1 / 2}=\frac{1}{2}\left(a_{i+1}+a_{i}\right)
$$

After substitution of the fluxes $\left(I^{\alpha}\right)_{i+1 / 2}$ and $\left(I^{\alpha}\right)_{i-1 / 2}$ into the difference equation (22), we obtain the difference scheme

$$
\begin{aligned}
\left(\Lambda^{\epsilon}\left(\beta_{\epsilon}\right)\left(\epsilon^{\alpha}\right)^{l+1}\right)_{i} & \equiv \frac{1}{h_{i}^{*}} A_{i}^{\epsilon}\left(\epsilon^{\alpha}\right)_{i-1}^{l+1}+\frac{1}{h_{i}^{*}} B_{i}^{\epsilon}\left(\epsilon^{\alpha}\right)_{i+1}^{l+1}-Q_{i}^{\epsilon}\left(\epsilon^{\alpha}\right)_{i}^{l+1} \\
& =-\left(\left(\epsilon^{\alpha}\right)_{i}^{l} / \tau_{l+1}+\left(N_{\alpha} T_{l}\right)_{i} / \tau_{\omega}\right)
\end{aligned}
$$


where

$$
\begin{aligned}
Q_{i}^{\epsilon} & =\frac{1}{h_{i}^{*}}\left(A_{i+1}^{\epsilon}+B_{i-1}^{\epsilon}\right)+\frac{1}{\tau_{l+1}}+\frac{2}{3} \frac{1}{\tau_{\omega}} \\
A_{i}^{\epsilon} & =\frac{2}{3} C^{\alpha}\left(\mu^{\alpha} T^{\alpha}\right)_{i-1 / 2}\left(\beta_{\epsilon}^{\alpha}\right)_{i-1 / 2} \frac{\exp \left(\left(\beta_{\epsilon}^{\alpha}\right)_{i-1 / 2}\right)}{h_{i}\left(\exp \left(\left(\beta_{\epsilon}^{\alpha}\right)_{i-1 / 2}\right)-1\right)}, \\
B_{i}^{\epsilon} & =\frac{2}{3} C^{\alpha}\left(\mu^{\alpha} T^{\alpha}\right)_{i+1 / 2}\left(\beta_{\epsilon}^{\alpha}\right)_{i+1 / 2} \frac{1}{h_{i+1}\left(\exp \left(\left(\beta_{\epsilon}^{\alpha}\right)_{i+1 / 2}\right)-1\right)} .
\end{aligned}
$$

The derived difference scheme is unconditionally monotone since its solution satisfies the conditions of the maximum principle $[2,4]$

$$
\left(\Lambda^{\epsilon}(\beta)\left(\epsilon^{\alpha}\right)^{l+1}\right)_{i} \leq 0, \quad A_{i}^{\epsilon}>0, \quad B_{i}^{\epsilon}>0,
$$

and

$$
Q_{i}^{\epsilon}=\frac{1}{h_{i}^{*}}\left(A_{i+1}^{\epsilon}+B_{i-1}^{\epsilon}\right)+D, \quad D=\frac{1}{\tau_{l+1}}+\frac{2}{3} \frac{1}{\tau_{\omega}} \geq 0 .
$$

The verification of the monotonicity conditions for the continuity equations, the error analysis of the proposed scheme, and its numerical realisation based on a semi-implicit algorithm are carried out in a manner similar to that proposed in [4]. In the next section we highlight several numerical results obtained with the proposed scheme.

\section{Numerical results}

We carried out a series of computational experiments with the developed model (15-18), and (5) by using the scheme constructed in the previous section. The major goal was to predict charge photo-responses for vertical field-effect phototransistors based on a GaAs structure. The gates of the structure were taken $2 \mu \mathrm{m}$ long, placed at equal distance $(2 \mu \mathrm{m})$ from the 
source and drain of the structure. The primary emphasis was given to the photo-charge response in the depleted region in the middle of the structure, between the gates. In this region conductive carries concentrations are low, and in our experiments we assume that $n_{c}=p_{c}=0$. The source-drain region was positively biased with $2 \mathrm{~V}$ potential applied to the drain at $x=0$. Our study focused on transport of photogenerated electrons with concentrations propagating towards the drain. The boundary conditions for concentrations (given in $\mathrm{cm}^{-3}$ ) were taken as $\delta n_{e}(0, t)=n_{0} / 10^{22}$ and $\partial\left(\delta n_{e}(L, t)\right) / \partial x=0$ with $n_{0}$ being the GaAs intrinsic concentration at lattice temperature $T_{l} \equiv$ $T_{0}=320 \mathrm{~K}$. The energy density and temperature boundary conditions were: $\epsilon^{e}(0, t)=N_{e}^{0} T_{0}, T^{e}(0, t)=T_{l}, \partial \epsilon^{e}(L, t) / \partial x=0, \partial T^{e}(L, t) / \partial x=0$. The value of $N_{e}^{0}=n_{e 0}+p_{e 0}$ was used with $p_{e 0}=n_{e 0}=10^{-3}$. Carrier mobilities were taken as $\mu=8000\left(300 / T_{0}\right)^{2 / 3} \mathrm{~cm}^{2} \mathrm{~V}^{-1} \mathrm{~s}^{-1}$. Finally, initial conditions were taken as $\delta n_{e}(x, 0)=n_{0} / 10^{22}, \epsilon^{e}(x, 0)=N_{e}^{0} T_{0}, T^{e}(x, 0)=T_{l}$, $\varphi(x, 0)=0$. The system of difference equations was solved in a semi-implicit manner by a generalisation of the algorithm proposed in [4] with predefined photogenerated carrier source term $G(x, t)=G_{0} \sin \left(\pi t / t_{g}\right)$, for $x_{l} \leq x \leq x_{r}$ with $x_{l}=0.85 \mu \mathrm{m}, x_{r}=1.15 \mu \mathrm{m}, t \leq t_{g}=10^{-19} \mathrm{~s}, G_{0}=10^{10} \mathrm{~cm}^{-3} \mathrm{~s}^{-1}$. For $x \leq x_{l}$ and $x \geq x_{r}(0 \leq t \leq T)$ as well as for $t \geq t_{g}(0 \leq x \leq L) G(x, t)=0$. As an example, we present spatio-temporal distributions of concentrations in Figure 1. Since we deal with a multiscale problem, the output is presented for subsequent computational time intervals (plots (a) and (b)) in order to better reflect two different processes involved. Note that theoretical estimates of the drift time in this problem is estimated as $t_{d}=L /\left(2\left|v_{d}\right|\right) \approx 1.4 \cdot 10^{-12}$ (with $v_{d}=\mu \partial \varphi / \partial x$ ), which is in a perfect agreement with the result of computations (see Figure 1b). Similar estimates hold for thermal drift time evaluated on the basis of an averaged value of temperature. 

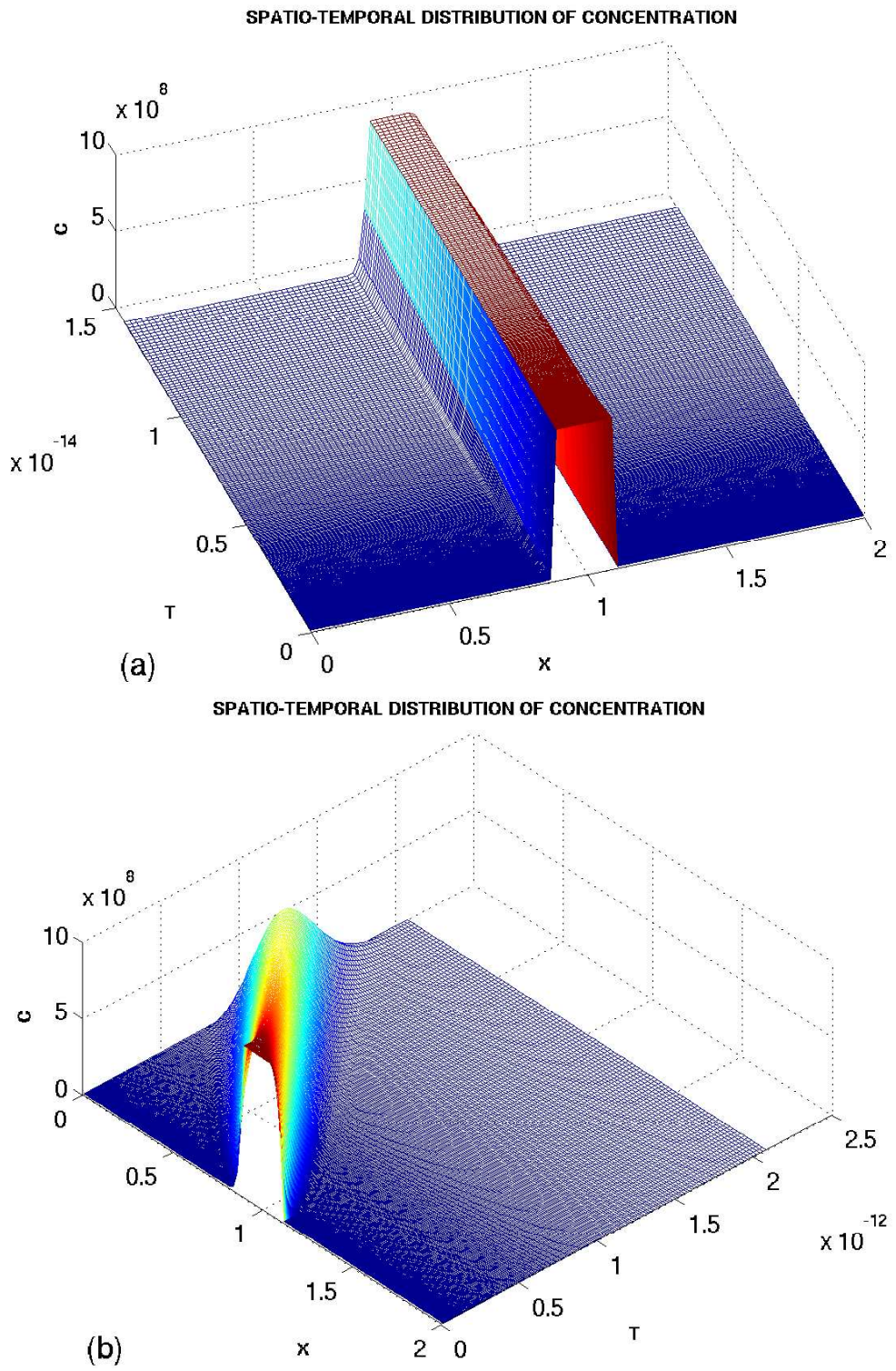

Figure 1: Concentration of photogenerated electrons at different time scales: (a) $0 \leq t \leq 1.4 \cdot 10^{-14}$; (b) $1.4 \cdot 10^{-14}<t<2.1 \cdot 10^{-12}$. 


\section{References}

[1] S. A. Abashkina, V. I. Korol'kov, J. S. Rimshans, Y. I. Skryl', T. S. Tabarov, Numerical Calculations of Transient Characteristics of GaAs Vertical Field-Effect Phototransistors, Semiconductors, 27(6): 524-529, 1993. C730, C733

[2] N. V. Karetkina, An unconditionally stable difference scheme for parabolic equations with first derivatives, Comp. Math. and Math. Physics, 20(1): 236-240, 1980. C739

[3] R. V. N. Melnik, and H. He, Modelling nonlocal processes in semiconductor devices with exponential difference schemes, J. Engineering Mathematics, 38: 233-263, 2000. C733, C737

[4] R. V. N. Melnik, J. Rimshans, Numerical Analysis of Fast Charge Transport in Optically Sensitive Semiconductors, Dynamics of Continuous, Discrete and Impulsive Systems, Series B, Proc. of the DCDIS-2003 on Engineering Applications and Computational Algorithms, 102-107, 2003. C733, C736, C739, C740

[5] D. M. Pooley, P. A. Forsyth, K. R. Vetzal, Numerical Convergence Properties of Option Pricing PDEs with Uncertain Volatility, IMA J. Numer. Anal., 23: 241-267, 2003. C731

[6] A. A. Samarskii, The theory of difference schemes, Marcel Dekker, N.Y., 2001. C737

[7] F. Qian et al., Indirect-Coupling UV-sensitive Photodector with High Electrical Gain, Fast Response, and Low Noise, Sensors and Actuators, 86: $66-72,2000 . \quad$ C 730

[8] J. Xu, L. Zikatanov, A monotone finite element scheme for convection diffusion equations, Math. Comp., 68: 1429-1446, 1999. C731 
[9] B. van Leer, Towards the Ultimate Conservative Difference Scheme, J. Comput. Phys., 135: 229-248, 1997. C731 